\title{
Metatypical basal cell carcinoma: from the primary tumour to a generalized metastatic process - description of diagnostics and combination therapy of an extremely rare skin cancer
}

\author{
Barbara Madej-Czerewonka ${ }^{\oplus}$, Agnieszka Korga-Plewko ${ }^{2}$ \\ ${ }^{1}$ Human Anatomy Department, Medical University, Lublin, Poland \\ ${ }^{2}$ Independent Medical Biology Unit, Medical University, Lublin, Poland \\ A - Research concept and design, B - Collection and/or assembly of data, C - Data analysis and interpretation, \\ $D$ - Writing the article, $E$ - Critical revision of the article, $F$ - Final approval of article

\begin{abstract}
Madej-Czerwonka B, Korga-Plewko A. Metatypical basal cell carcinoma - from the primary tumour to a generalized metastatic process - a description of diagnostics and combination therapy of an extremely rare skin cancer. Ann Agric Environ Med. 2022; 29(1): 152-156. doi: $10.26444 /$ aaem/134660
\end{abstract}

\section{Abstract}

Introduction. Basal cell carcinoma (BCC) is the most common form of skin cancer. The hallmarks of this carcinoma are the absence of distant metastases and local malignancy. Metatypical basal cell carcinoma (MTBBC) is rare variant that it is considered to be a more aggressive form, with a higher potential for metastases and recurrence. Probably due to its very rare occurrence, data on pathogenesis, course and treatment are inconsistent.

Case report. An unusual and very aggressive course of MTBBC with multiple metastases is prtesented. Repeated histopathological evaluation shows the diagnostic difficulties in this type of tumour. Therapeutic attempts, including targeted therapy with vismodegib, were unsuccessful.

Conclusions. This case showed that it should always be taken into account that each case of diagnosed BCC may turn out to be metatypical variant with a much more aggressive course and worse prognosis. For this reason, intensive follow-up after a completed treatment is recommended.

\section{Key words \\ skin cancer, metastases, metatypical basal cell carcinoma, vismodegib}

\section{INTRODUCTION}

Skin cancer is the most common type of all malignant tumours in Caucasians [1], with cutaneous basal cell carcinoma (cBCC) accounting for $80 \%$ of all skin malignancies [1]. cBCC is described as a locally invasive malignant tumour which rarely metastasises or causes the death of the patient. It is characteristic for $\mathrm{BCC}$ to grow locally, infiltrate surrounding tissues, and destroy deeper structures, i.e. cartilage or bone. The progression of $\mathrm{CBCC}$, due to its prevalence mainly in the face and neck skin, can result in significant aesthetic and functional defects in patients [2].

cBCC poses a considerable challenge to otorhinolaryngology (head and neck) surgeons [3]. Global statistics indicate that the highest incidence rate of $\mathrm{CBCC}$ is observed in Australia with the annual number of cases reaching approximately 884 per 100,000 of people. On the other hand, some regions of Africa have the lowest incidence rate with under 1 per 100,000 citizens [4]. On average, European countries report from 44 up to 128 new cases per 100,000 of people each year [5]. Lifetime risk of developing cBCC in Caucasians exceeds $30 \%$ [6]. In recent years an increasing incidence of $\mathrm{CBCC}$ has been observed. Males and elderly people are more prone to develop cBCC, and men are significantly more likely than

Address for correspondence: Agnieszka Korga-Plewko, Independent Medical Biology Unit, Medical University, Lublin, Poland

E-mail: agnieszka.korga@umlub.pl

Received: 25.01.2021; accepted: 19.03.2021; first published:09.04.2021 women to present cBCC $(1.5-2: 1)$ [7]. In over $50 \%$ of cases, cBCC is diagnosed in patients aged $50-80$.

Apart from advanced age and male gender, a crucial cBCC risk factor is an excessive exposure to ultraviolet radiation. Although the pathogenesis of this process is still poorly understood it has been proven that sunscreen use does not necessarily translate into a lower $\mathrm{cBCC}$ incidence rate [8]. Other risk factors include: actinic keratosis, side-effects of occupational exposure to $\mathrm{x}$-ray radiation, burn scars or scars being the result of chronic skin inflammations, and human papillomavirus (HPV) infection [9].

Preliminary diagnosis is established on the basis of clinical and physical examination. Macroscopic characteristics help to detect $\mathrm{cBCC}$ which may be classified in three main clinical subtypes: nodular, superficial, and morpheaform. Each lesion examination should be performed with the use of a dermatoscope. An accurate $\mathrm{CBCC}$ diagnosis should be established on the basis of a histopathological examination of the excised tissue. It is crucial to evaluate the measurements of the lesion, including its infiltration depth and surgical margins. In the case of any diagnostic uncertainty, it is possible to perform an immunohistochemical examination in order to differentiate between a $\mathrm{CBCC}$ and a squamous cell carcinoma (SCC) [10].

In the case of local cancer progression it is advisable to additionally implement such imaging techniques as computed tomography scan (CT scan) and/or magnetic resonance imaging (MRI). Histological subtype of cancer, the clinical 
stage of the cancer, and the overall health status of the patient are decisive for further treatment strategies $[10,11]$.

\section{CASE STUDY}

In 2002, onset of disease was reported by a 37-year-old male who attended a surgical oncology clinic to consult a tiny lump observed in the upper lip. An excisional biopsy was performed followed by an upper lip reconstruction. The excised tissue was sent for histopathological examination. The results showed ulcerative BCC. Surgical margins were tumour-free. The patient was scheduled for regular check-ups at an outpatient surgical oncology centre.

In 2012, the man was concerned about his chronic fatigue, decline in his physical efficiency, and headaches radiating downward to the neck. Firstly, the patient underwent a series of blood tests on an outpatient basis (blood count, liver and kidney function tests). The test results showed no abnormalities. A head MRI was performed which helped to detect only slightly enlarged pericerebral fluid spaces in the parietal lobes. A neck ultrasound examination followed, chich showed a heterogeneous tissue mass with relatively well-circumscribed margins, measuring $20 \times 19 \mathrm{~mm}$, located in the right mandibular angle. The intramuscular lesion resulted in posterior pressure over the sternocleidomastoid muscle. Single lymph nodes were visible on examination, the most prominent one measuring approx. $18 \times 4 \mathrm{~mm}$. On the left, noticeable lymph nodes measured $15 \times 6 \mathrm{~mm}$. A CT scan of the neck was performed which confirmed the presence of a tumour and its infiltration of the right internal jugular vein.

After completion of the abov-ementioned examinations the patient was referred to the department of otolaryngology in order to undergo a surgical treatment of the neck tumour. A pre-operative CT scan of the chest was performed. The results showed focal lesions suggestive of metastases in the lung parenchyma. In the right lung, the following lesions were detected: in the superior segment (S VI) a lesion measuring $13 \mathrm{~mm}$, in its medial segment (S V) $-7 \mathrm{~mm}$, and in its posterior basal segment (S X) - $22 \mathrm{~mm}$. In the left lung, lesions were found in the anterior segment (S III), a lesion measuring $22 \mathrm{~mm}$ and in the superior segment (S VI) $-10 \mathrm{~mm}$. No pathologically enlarged lymph nodes were observed. An ultrasound examination of the abdominal cavity detected no pathological changes.

After a series of diagnostic examinations the patient underwent the following surgery: the skin and subcutaneous tissue were incised along sternocleidomastoid muscle reaching to the neck tumour which had infiltrated the right internal jugular vein, scalene muscles, cervical plexus and accessory nerve. A tumour sample was taken for intraoperative histopathological evaluation (Fig. 1). The following results were obtained: keratinizing SCC (G2), infiltrating muscle and adipose tissue.

The lesion was removed entirely including cervical lymph nodes, an internal jugular vein, and a sternocleidomastoid muscle. Cervical plexus and accessory nerve were cut.

A postoperative histopathological evaluation of 12 lymph nodes recognised two metastases (cancerous infiltration of perinodal connective and adipose tissue), and the patient was referred to an oncology centre for further treatment. The unusual course of the disease made the specialists conduct histopathological evaluation for the second time. Paraffin-

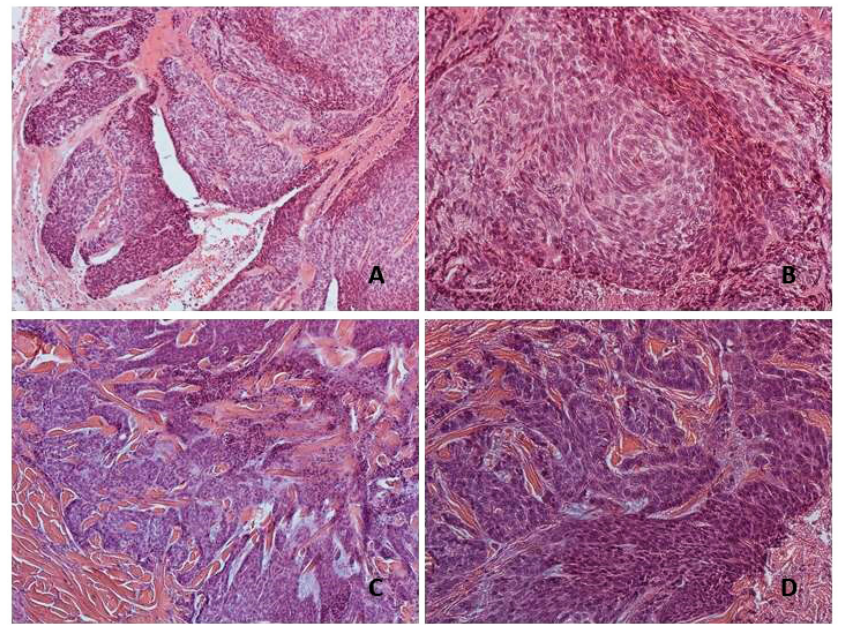

Figure 1. Metatypical basal cell carcinoma - neck metastatic tumour $(H+E ; A, C$ 100x, B,D 200x)

embedded tissue blocks from the upper lip and neck tumour were re-examined. The findings were analysed again and showed desmoplastic infiltrative BCC.

During the next hospitalisation, the patient had a wedge resection of the right lung due to the above-mentioned metastases. Both metastatic foci were located beneath the pleura and had infiltrated the pulmonary pleura. On microscopic examination, specimens of lung parenchyma were analysed, chich showed metastases from skin appendages. Morphological assessment of these metastases gave very similar results to the findings obtained after upper lip and neck tumour examinations. In the case of the latter, a metatypical basal cell carcinoma (MTBCC) or basal cell (trichoblastic) carcinoma were suspected. Thirty days later, the patient was admitted for the next stage of the surgery. Left-sided thoracotomy and a wedge resection of four lesions were performed. On microscopic examination, metastatic foci from skin appendages were observed, located beneath the pleura.

A PET-CT scan was performed in order to find the primary tumour focus. Markedly increased 18FDG uptake was noted in the post-operative area around C6 vertebra, in the anterior segment (S III) of the left lung, above the diaphragm, in the anterior basal segment (S VIII), and in the lateral basal segment (S IX) of the right lung. Physiological tracer uptake was observed within the abdominopelvic cavity. Increased tracer uptake was noted in the skeletal system, i.e. in the 5th right rib and in the 6th left rib (Fig. 2).

The next stage of treatment required the patient to undergo a chemotherapy. Within four months he received in total 10 cycles of AP3 chemotherapy, a dose of Epi ADM 65 mg once up to three times a day and a dose of CBDCA $345 \mathrm{mg}$ once or twice a day. A month after the last chemotherapy cycle, the patient was admitted to hospital due to side-effects such as grade 3 leukopenia and grade 4 thrombocytopenia.

Two months after chemotherapy ended, the patient underwent another PET-CT scan in order to evaluate changes in the skeletal system. The findings showed a hypodense lesion around the thyroid cartilage, adjacent to the right common carotid artery, situated in the superior pole of the right thyroid lobe and measuring $12 \times 16 \times 14 \mathrm{~mm}$. The scan indicated tumour recurrence. Skeletal system presented no abnormalities in glucose uptake. 

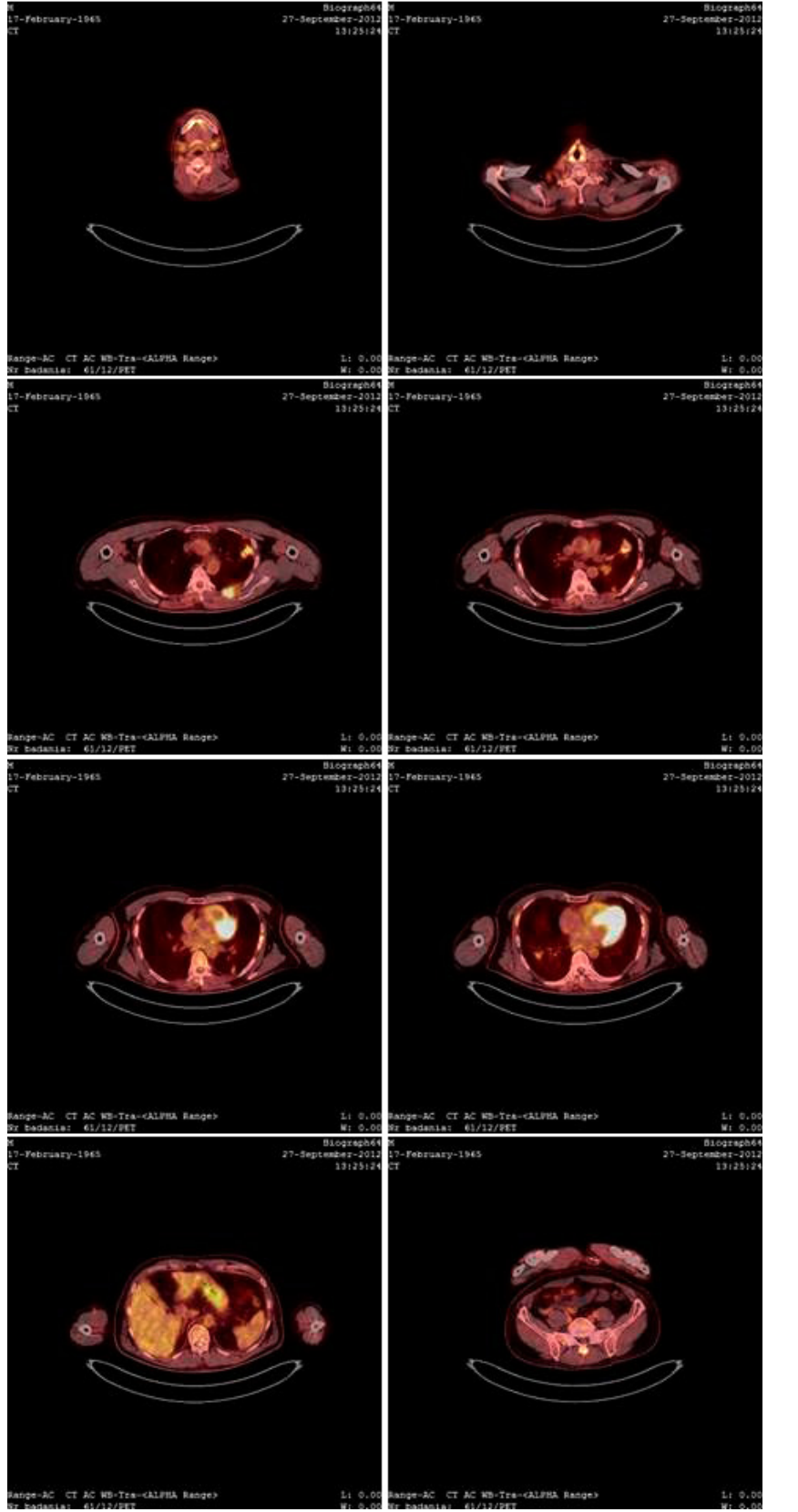

Figure 2. $P E T-C T$ scan showing general cancer recurrence in the course of a metatypical basal cell carcinoma of the upper lip

The next stage of patient's treatment involved complete tumour removal and post-operative radiotherapy. A radical intensity-modulated radiation therapy (IMRT) was performed. Patient's right-sided cervical lymph nodes and post-operative area were treated in total with $66 \mathrm{~Gy}$ dose. On histopathological examination SCC was recognised.

Follow-up CT scans of the chest and abdominal cavity were performer chich showed newly-formed micronodular lesions in the right and left lungs. No enlarged lymph nodes were observed.

The patient was qualified for surgery. A laser-assisted resection of the lesions present in the left lung was performed. Histopathological examination of the excised tumour recognised SCC. After two months, another laserassisted resection of four lesions located in the right lung was performer.
Six months later, a PET-CT scan was performed in order to evaluate the current progression of the disease. The findings showed two tumours of malignant potential, located in the posterior basal segment (S X) and in the anterior segment (S III) of the left lung. A CT scan, performed a month later, found significant tumour progression in comparison to the results obtained five months earlier. In the left lung, in its anterior segment (S III) tumour diameter increased by $4 \mathrm{~mm}$, in the anterior basal segment (S VIII) - by $4 \mathrm{~mm}$, in the posterior basal segment ( $\mathrm{X}$ ) - by $5 \mathrm{~mm}$, whereas in the right lung, in its posterior segment (S II), tumour diameter increased by $4 \mathrm{~mm}$ and in its lateral segment (S IV) - by $2 \mathrm{~mm}$. Having analysed the results of imaging tests the patient was recommended to undergo another cycle of chemotherapy, which he declined.

Due to the unusual course of the disease, paraffinembedded tissue blocks from the upper lip and neck tumours underwent histopathological evaluation for the third time. The following results were obtained:

- in the upper lip specimen, an infiltration of non-small-cell carcinoma was observed, its morphological features were similar to a metatypical basal cell carcinoma (MTBCC) or basosquamous carcinoma that incorporates the features of BCC, with partial differentiation towards keratinizing SCC in the centre of the lesion;

- the neck tumour specimen and a sample of lesions located in the lungs showed structures resembling a non-small-cell carcinoma with morphological features similar to those characterising metastases of a MTBCC.

After analysis of the above-mentioned findings, the patient was qualified for a therapy with the use of Vismodegib, a Hedgehog (Hh) signaling pathway inhibitor, at a daily dose of $150 \mathrm{mg}$. After a six-month Vismodegib treatment, a CT scan of the chest was performed in order to evaluate the metastases in the lungs. In the right lung, in its lateral segment (S IV), a tumour regression from $27 \mathrm{~mm}$ to $12 \mathrm{~mm}$ was observed. In the left lung, in its posterior basal segment (S X), tumour regression from $17 \mathrm{~mm}$ to $5 \mathrm{~mm}$ was noted.

In the thirteenth month of Vismodegib therapy, the patient was admitted to hospital due to severe fatigue. CT scans of the head, neck and chest were performed. Results:

- a newly-formed lesion measuring $11 \times 10 \mathrm{~mm}$ was detected in the submandibular space;

- in the right lung, in its lateral segment (S IV), an increase in tumour diameter from 12 to $20 \mathrm{~mm}$ was observed; in its posterior segment (S II), an increase in tumour diameter from $9 \mathrm{~mm}$ to $17 \mathrm{~mm}$ was noted;

- in the left lung, in its posterior segment (S II), an increase in tumour diameter from $6 \mathrm{~mm}$ to $10 \mathrm{~mm}$ was detected, in the anterior segment (S III), an increase in tumour formed lesion measuring $8 \mathrm{~mm}$ was observed, in the anterior basal segment (S VIII) a 28-mm tumour was detected, previously the lesion was not traceable.

Over the next nine months the patient underwent followup CT scans of the neck and chest (every two months) which indicated a gradual progression of the lesions.

At the same time, the patient underwent another surgical operation for removal of a tumour located in the infrahyoid muscles (Fig. 3). The patient received post-operative intravenous chemotherapy with 5-fluorouracil $500 \mathrm{mg}$, four cycles according to guidelines. 


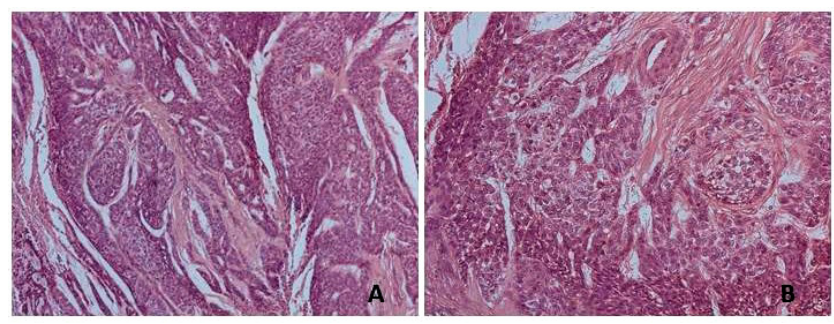

Figure 3. Metatypical basal cell carcinoma - tumour located in the infrahyoid muscles $(\mathrm{H}+\mathrm{E} ; \mathrm{A} 100 \times, \mathrm{B} 200 \times)$

A follow-up CT scan showed a newly-formed metabolicallactive lesion located to the left of the supraclavicular fossa. The patient was qualified for radiotherapy. Stereotactic body radiation therapy (SBRT) was administered. The patient received in total a dose of 30 Gy in six fractions every two days. Six months after the radiotherapy ended, follow-up CT scans of the neck and chest were performed. In the left lung, in its anterior segment (S III), a decrease from $10 \mathrm{~mm}$ to $8 \mathrm{~mm}$ in one of the tumour diameters was observed. Measurements of other metastases remained the same in comparison to the results of previous scans. A newly-formed lesion measuring $30 \times 12 \mathrm{~mm}$ was detected on the right side of the base of the neck, sideways from the thyroid gland. Hypodense lesions, suspected metastases, were found in the left and right kidneys. Having consulted a laryngologist the patient was not qualified for surgery due to the fact that a radical removal of the lesion was not possible. The consecutive stages of treatment involved palliative care.

The patient was hospitalised occasionally due to fatigue, dehydration, severe abdominal and cervical pain inthe course of cancer. The patient experienced cachexia and had massive neck ulceration (Fig. 4), anaemia ( $\mathrm{Hb} 8 \mathrm{~g} / \mathrm{dl}$ ) and kidney failure. During hospitalisation, the patient underwent fluid therapy, blood transfusion therapy and a local treatment of the neck ulcers. The patient died in 2018, fifteen years after the first diagnosis.

\section{DISCUSSION}

To the authors' knowledge, there is scant information in the literature on MTBCC, and methods of treatment are not standardized. Bearing in mind that five-year survival in patients diagnosed with MTBCC accounts for only $10 \%$, it poses a crucial clinical challenge for specialists $[12,13]$. MTBCC is a skin cancer sharing features of both SCC and BCC. Morphological features of MTBCC resemble BCC, yet its clinical characteristics include significantly higher malignancy and a tendency to metastasize [14]. Establishing an accurate histopathological diagnosis of MTBCC is extremely difficult. As the presented case study shows, it was possible to detect MTBCC only after histopathological analysis of the specimens had been repeated several times. The most common location for distant metastases of MTBCC are lungs, bones and liver [14].

Basic treatment for a locally advanced cancer includes surgical removal of the lesion. When assessing the width of the surgical margins one should take into account both the radical character of the surgery and satisfactory aesthetic effects of the operation. Sometimes, this poses a significant challenge for the surgeon since macroscopic tumour borders are not clear. For tumours with a high risk of recurrence, the surgical margin is estimated at $10 \mathrm{~mm}$ at least [15]. Mauro Tallaro et al. proved in their study that in a significant number of specimens subjected to evaluation, the surgical margins were positive [16].

Patients coping with metastatic cancer who have undergone surgical treatment and adjuvant radiotherapy are advised to include pharmacotherapy. Medicines approved for such clinical cases are Hedgehog (Hh) signaling pathway

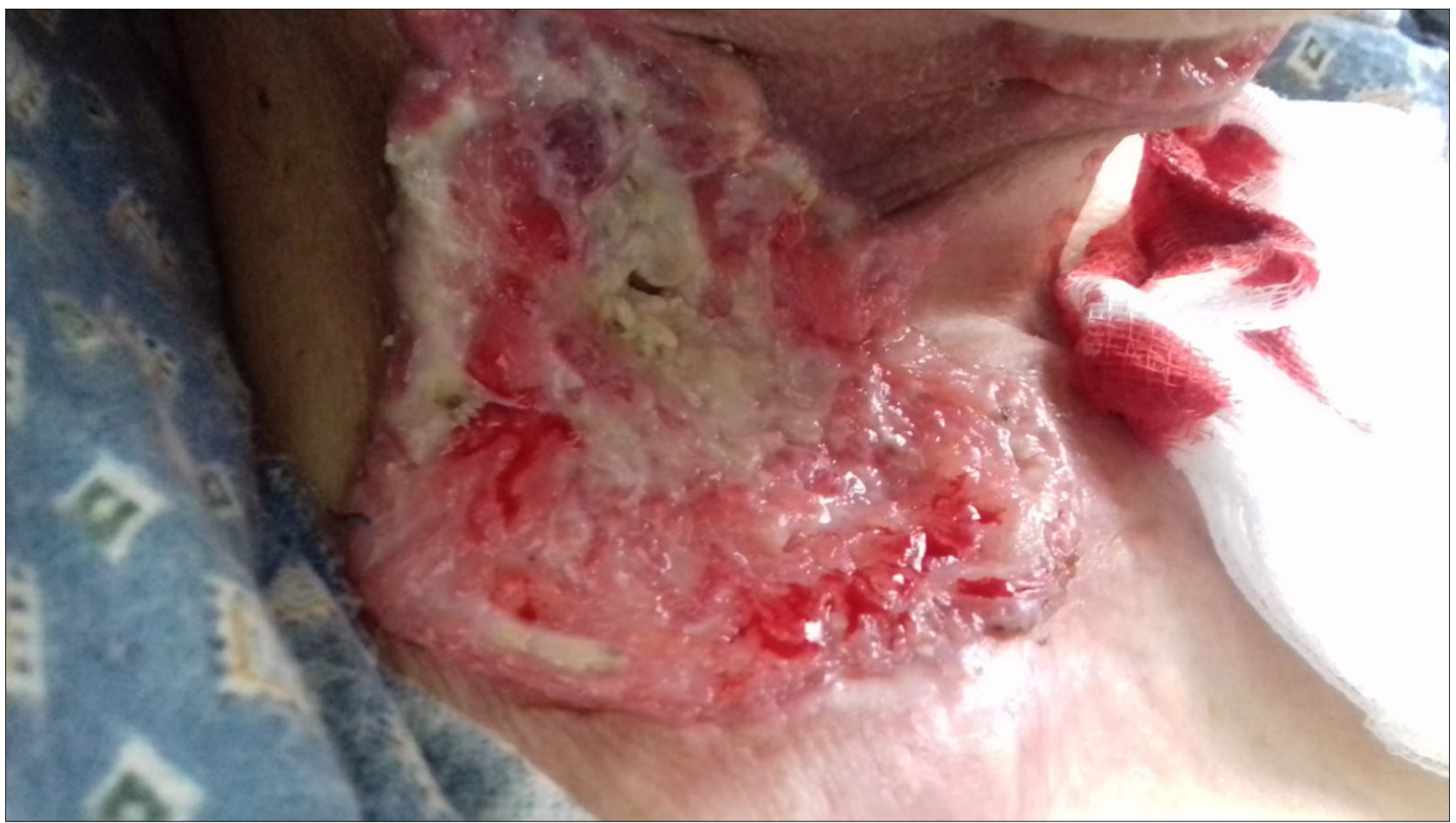

Figure 4. Neck ulceration in the end stage of the disease 
inhibitors. The Hh signal transduced by Smo protein results in activation and localisation of the Gli transcription factors in the cell nucleus, as well as induction of Hh target genes. Vismodegib is a pharmacologic agent and Smo inhibitor chich binds to the Smo protein and inhibits its function. The drug was approved for adults diagnosed with BCC with distant metastases, as well as for patients with locally advanced tumour who cannot have surgery or radiotherapy. Vismodegib should be administered at a dose of $150 \mathrm{mg}$ per day. According to the data found in specialised literatur, although a high responsiveness to the Vismodegib treatment is observed, $25 \%$ of patients may become resistant to it during the first year of therapy [17].

Clinical findings concerning the use of chemotherapy are rarely reported. Systemic chemotherapy usually uses cisplatin, doxorubicin, 5-fluorouracil [14, 18].

\section{CONCLUSIONS}

MTBCC remains a significant problem for head and neck surgeons. When such a subtype of cancer is detected, the surgeon should consider extending the resection margins around the primary lesion. However, as shown in the presented case, its histopathological diagnosis may raise a number of doubt, and this rare subtype of BCC may not always be diagnosed immediately. Therefore, it should always be taken into account that each case of diagnosed BCC may turn out be its metatypical variant with much more aggressive course and worse prognosis. For this reason, after completed treatment, intensive follow-up is recommended and it is advisable to apply the full three months.

Informed Consent Statement. Informed written consent for publication was not requested from the local Ethics Committee, since no data in the case description could potentially or positively reveal the identity of the patient.

\section{REFERENCES}

1. Rutkowski P. Owczarek, W. Guidelines for diagnostic and therapeutic management: skin carcinomas. Oncol Clin Pract. 2018; 14(3): 129-147. https://doi.org/10.5603/OCP.2018.0019
2. De Giorgi V, Savarese I, Gori A, et al. Advanced basal cell carcinoma: when a good drug is not enough. J Dermatolog Treat. 2020; 31(6): 552-553. https://doi.org/10.1080/09546634.2018.1542481

3. Castanheira A, Boaventura P, Pais Clemente M, et al. Head and neck cutaneous basal cell carcinoma: what should the otorhinolaryngology head and neck surgeon care about? Acta Otorhinolaryngol Ital. 2020; 40(1): 5-18. https://doi.org/10.14639/0392-100X-2245

4. Chinem VP, Miot HA. Epidemiology of basal cell carcinoma. An Bras Dermatol. 2011; 86(2): 292-305. https://doi.org/10.1590/s036505962011000200013

5. Lomas A, Leonardi-Bee J, Bath-Hextall F. A systematic review of worldwide incidence of nonmelanoma skin cancer. Br J Dermatol. 2012; 166(5): 1069-80. https://doi.org/ 10.1111/j.1365-2133.2012.10830.x

6. Mori S, Cordova M, Nehal KS, et al. Basal cell carcinoma: Epidemiology; pathophysiology; clinical and histological subtypes; and disease associations. J Am Acad Dermatol. 2019; 80(2): 303-317. https://doi. org/10.1016/j.jaad.2018.03.060

7. Richmond-Sinclair NM, Pandeya N, Ware RS, et al. Incidence of basal cell carcinoma multiplicity and detailed anatomic distribution: longitudinal study of an Australian population. J Invest Dermatol. 2009; 129(2): 323-8. https://doi.org/10.1038/jid.2008.234

8. Sánchez G, Nova J, Rodriguez-Hernandez AE, et al. Sun protection for preventing basal cell and squamous cell skin cancers. Cochrane Database Syst Rev. 2016; 7(7): CD011161. https://doi.org/10.1002/14651858. CD011161.pub2

9. Stern RS. PUVA Follow-Up Study. The risk of squamous cell and basal cell cancer associated with psoralen and ultraviolet A therapy: a 30-year prospective study. J Am Acad Dermatol. 2012; 66(4): 553-62. https:// doi.org/10.1016/j.jaad.2011.04.004

10. Rutkowski P, Jassem J, Krzakowski M. Złośliwe nowotwory skóry. 2nd edition. Gdańsk: ViaMedica; 2014.

11. Berking C, Hauschild A, Kölbl O, et al. Basal cell carcinoma-treatments for the commonest skin cancer. Dtsch Arztebl Int. 2014; 111(22): 389-95. https://doi.org/10.3238/arztebl.2014.0389

12. Saàdani CH, Baybay H, ElMahi H, et al. Basosquamous Carcinoma: Report of Two Cases and Review of Literature. Int J Cancer Clin Res. 2018; 5(1): 093. https://doi.org/10.23937/2378-3419/1410093

13. Tchernev G, Ananiev J, Cardoso JC, et al. Metatypical Basal cell carcinomas: a successful surgical approach to two cases with different tumor locations. Maedica (Bucur). 2014; 9(1): 79-82.

14. McCusker M, Basset-Seguin N, Dummer R, et al. Metastatic basal cell carcinoma: prognosis dependent on anatomic site and spread of disease. Eur J Cancer. 2014; 50(4): 774-83. https://doi.org/10.1016/j. ejca.2013.12.013

15. Rutkowski P, Owczarek W. Biblioteka Chirurga Onkologa. Dermatochirurgia. Gdańsk: Via Medica; 2018. p. 12.

16. Tarallo M, Cigna E, Frati R, et al. Metatypical basal cell carcinoma: a clinical review. J Exp Clin Cancer Res. 2008; 27(1): 65. https://doi. org/10.1186/1756-9966-27-65

17. Frampton JE, Basset-Séguin N. Vismodegib: A Review in Advanced Basal Cell Carcinoma. Drugs. 2018; 78(11): 1145-1156. https://doi. org/10.1007/s40265-018-0948-9

18. Shukla S, Khachemoune A. Reappraising basosquamous carcinoma: a summary of histologic features, diagnosis, and treatment. Arch Dermatol Res. 2020; 312(9): 605-609. https://doi.org/10.1007/s00403020-02058-1 